\title{
Recurrent attacks of acute hepatic porphyria: major role of the chronic inflammatory response in the liver
}

C. Schmitt ${ }^{1,2,3}$, H. Lenglet ${ }^{2,3}$, A. Yu ${ }^{4}$, C. Delaby ${ }^{5}$, A. Benecke ${ }^{6,7}$, T. Lefebvre ${ }^{1,2,3}$, P. Letteron ${ }^{2}$, V.

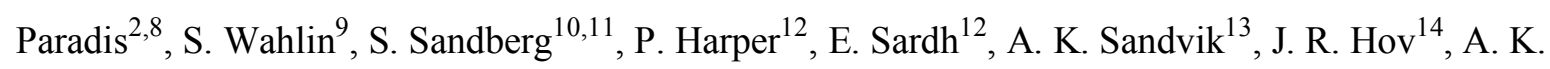
Aarsand $^{10,11}$, L. Chiche ${ }^{15}$, C. Bazille ${ }^{16}$, J.-Y. Scoazec ${ }^{17}$, J. To-Figueras ${ }^{18}$, M. Carrascal ${ }^{19}$, J. Abian ${ }^{19}$, A. Mirmiran ${ }^{2,3}$, Z. Karim ${ }^{2,3}$, J.-C. Deybach ${ }^{1,2,3}$, H. Puy ${ }^{1,2,3}$, K. Peoc'h ${ }^{2,3,20}$, H. Manceau ${ }^{2,3,20} \&$ L. Gouya $^{1,2,3}$

\footnotetext{
${ }^{1}$ From the Centre Franc"ais des Porphyries, Hospital Louis Mourier, Assistance publique-Hospitaux de Paris (AP-HP), Colombes; ${ }^{2}$ Centre de Recherche sur 1'Inflammation (CRI), UMR1149 INSERM, Universite Paris Diderot, site Bichat; ${ }^{3}$ Laboratoire d'excellence, GR-Ex, Paris; ${ }^{4}$ STIM CNRS ERL 7368, Physiologie des Cellules Cardiaques et Vasculaires, Tours; ${ }^{5}$ Laboratory for Clinical Biochemistry and Proteomics, Institute for Regenerative Medicine and Biotherapy (IRMB), CHU de Montpellier and Universite Montpellier, Montpellier; ${ }^{6}$ Centre National de la Recherche Scientifique, Institut des Hautes Etudes Scientifiques, Bures-sur-Yvette, France ${ }^{7}$ Center for Innate Immunity and Immune Disease (CIIID), University of Washington School of Medicine, Seattle, WA, USA; ${ }^{8}$ DHU Unity, Pathology Department, Hospital Beaujon, AP-HP, Clichy, France; ${ }^{9}$ Department of Gastroenterology and Hepatology, Karolinska University Hospital and Karolinska Institutet, Stockholm, Sweden; ${ }^{10}$ Laboratory for Clinical Biochemistry, Norwegian Porphyria Centre (NAPOS), Haukeland University Hospital; ${ }^{11}$ Department of Global Health and Primary Health Care, University of Bergen, Bergen, Norway; ${ }^{12}$ Porphyria Centre Sweden, Centre for Inherited Metabolic Diseases, Karolinska Institutet, Karolinska University Hospital, Stockholm, Sweden; ${ }^{13}$ Department of Gastroenterology and Hepatology, St. Olav's University Hospital, Trondheim; ${ }^{14}$ Department of Transplantation Medicine, Norwegian PSC Research Center and Section of Gastroenterology, Oslo University Hospital and University of Oslo, Oslo, Norway; ${ }^{15}$ Centre Hospitalier Universitaire Bordeaux, Chirurgie Hepatobiliaire et Pancreatique, Maison du Haut Le ve que, Bordeaux; ${ }^{16}$ Service d'Anatomie Pathologique, Centre Hospitalo-Universitaire de Caen, Caen; ${ }^{17}$ Service d'anatomopathologie, Institut Gustave Roussy, Villejuif, France; ${ }^{18}$ Biochemistry and Molecular Genetics Department, Hospital Cl inic, IDIBAPS, University of Barcelona; ${ }^{19} \mathrm{CSIC} / \mathrm{UAB}$ Proteomics Laboratory, IIBB-CSIC, IDIBAPS, Barcelona, Spain; and ${ }^{20}$ Laboratory for Clinical Biochemistry, Ho^pital Beaujon, AP-HP, Clichy, France
} 


\section{Abstract:}

Background.

Acute intermittent porphyria (AIP) is an inherited disorder of haem metabolism character- ized by life-threatening acute neurovisceral attacks due to the induction of hepatic d-aminolevulinic acid synthase 1 (ALAS1) associated with hydroxymethylbilane synthase (HMBS) deficiency. So far, the treatment of choice is hemin which represses ALAS1. The main issue in the medical care of AIP patients is the occurrence of debilitating recurrent attacks.

Objective.

The aim of this study was to determine whether chronic hemin administration contributes to the recurrence of acute attacks.

Methods.

A follow-up study was conducted between 1974 and 2015 and included 602 French AIP patients, of whom 46 had recurrent AIP. Moreover, we studied the hepatic transcriptome, serum proteome, liver macrophage polarization and oxidative and inflam- matory profiles of Hmbs' mice chronically treated by hemin and extended the investigations to five explanted livers from recurrent AIP patients.

Results.

The introduction of hemin into the pharma- copeia has coincided with a 4.4-fold increase in the prevalence of chronic patients. Moreover, we showed that both in animal model and in human liver, frequent hemin infusions generate a chronic inflammatory hepatic disease which induces HO1 remotely to hemin treatment and maintains a high ALAS1 level responsible for recurrence.

Conclusion. Altogether, this study has important impacts on AIP care underlying that hemin needs to be restricted to severe neurovisceral crisis and suggests that alternative treatment targeting the liver such as ALAS1 and HO1 inhibitors, and antiinflammatory therapies should be considered in patients with recurrent AIP. 


\section{Introduction}

Acute intermittent porphyria (AIP), the most com- mon acute hepatic porphyria, is an autosomal dominant disease that occurs as a result of a $50 \%$ decrease in the activity of hydroxymethylbilane synthase (HMBS), the third enzyme of the haem biosynthesis pathway [1]. AIP is characterized by the intermittent occurrence of neurovisceral attacks, which typically consist of severe abdomi- nal pain and may include hypertension, tachycar- dia, confusion, motor paralysis and seizures and may be life-threatening. AIP is estimated to affect 5.4 per million people in European countries [2]. The pathogenesis of the acute attacks is hypothe- sized to result from an overproduction of a hepatic neurotoxic metabolite, presumably d-aminolevuli- nic acid (ALA) [3, 4]. This notion is consistent with the correction of the disease by liver transplanta- tion in patients with severe AIP [5-9]. Moreover, when domino liver transplantations were per- formed, the recipients developed biological and clinical acute porphyric attacks [10]. Altogether, these observations strongly support the hypothesis that the liver is the main organ involved in the pathogenesis of acute attacks.

The induction of the acute attacks is related to environmental or hormonal factors, such as cyto- chrome P450-inducing drugs, fasting, hormonal fluctuations during the menstrual cycle, infection or inflammation. These factors either directly induce the first enzyme of haem biosynthesis, d-aminolevulinic acid synthase 1 (ALAS1), or increase the demand for haem synthesis in the liver and subsequently suppress the negative feed- back of ALAS1. Carbohydrate loading, usually with intravenous glucose, may be an effective treatment in patients with noncomplicated acute attacks (without peripheral and central neurological signs or hyponatremia). Glucose administration, together with the resulting secondary increase in insulin, represses ALAS1 transcription via the inhibition of the transcriptional coactivator PGC- 1alpha [11]. However, intravenous human hemin administration, which restores the free haem pool and the negative feedback of ALAS1, is, so far, the treatment of choice of acute attacks of porphyria. Early human hemin infusion is a highly effective treatment [12].

Acute intermittent porphyria is a low penetrance disorder. The penetrance has been estimated to be $23 \%$ in European countries [2]. Most symptomatic AIP patients experience a limited number of acute attacks in a lifetime, and the attacks often occur once and in all cases, the attacks are associated with clearly identified precipitating factors. How- ever, a limited but significant number of patients become disabled by repetitive acute episodes with or without concomitant precipitating factors. These patients are treated with repeated haem infusions either to treat or to prevent acute attacks [13-17]. Some of these patients will even require monthly to weekly haem infusions. In Europe, approximately 5\% of AIP patients suffer from recurrent attacks [2]. In addition to recurrent acute attacks, these chronic patients encounter many medical side effects and have markedly impaired quality of life [18]. The large majority of these patients respond well to haem therapy, but long-term treatment may induce a dependency on exogenous haem, a need for indwelling access due to alterations in the superficial venous system, a rare but severe thrombotic event $[15,16]$.

In this study, we followed up a cohort of 602 symptomatic patients, of whom 46 had recurrent AIP, to document the natural history of the occur- rence of chronicity in AIP.

To elucidate the role of hemin in the recurrence of AIP attacks, we studied the hepatic transcriptome, serum proteome, liver macrophage polarization and oxidative and inflammatory profiles of an AIP mouse model which reproduces recurrent neurovisceral crisis treated by hemin. Moreover, thanks to the European Porphyria Network (EPNET), we extended the investigations to five human liver explants. We showed that chronic haem adminis- tration induces liver iron overload and sustains a strong oxidative stress response, which is known to activate haem oxygenase 1 (HO1). Surprisingly, in the AIP mouse model and human liver explants, we demonstrate that regular hemin administra- tion induces chronic hepatic inflammation responsible of a $\mathrm{HO} 1$ induction remotely to haem administration that triggers ALAS1 and thus recurrence.

\section{Materials and methods}


All animal experiments were performed according to procedures approved by the Bichat-Debre ethics committee. Animals were housed in a controlled environment with a 12-h light-dark cycle with free access to water and food. Animals were female C57BL/6 Hmbs' mice [19], 8 weeks old, weighing 20-25 g. Drugs (phenobarbital $100 \mathrm{mg} \mathrm{kg}^{1}$, haem arginate $8 \mathrm{mg}$ $\mathrm{kg}^{1}$ ) were injected intraperitoneally. For each group, we treated six female mice (Fig. 2a). Every week, we treated the mice with phenobarbital for 3 days followed by a 2-day treatment with hemin (PB/HA group) or excipients (arginine, ethanol and propy- lene glycol) (PB/Excipients group) or saline (PB/ Saline group). These treatments were repeated for 8 weeks. Another control group consisted of a noninduced group of mice injected everyday with saline (Saline group). When animals were sacri- ficed, liver tissues and blood samples were har- vested.

Microarray analysis of gene expression in the mouse liver

The mouse liver transcriptome was analysed using Mouse Genome Survey Arrays v2.0 (Applied Biosystems, Foster City, CA, USA), containing 32996 probes (60-mer oligonucleotide), which represented a set of 32381 annotated mouse genes. A total of 12 independent experiments were performed for the two different biological condi- tions (PB/HA and PB/Saline groups). Transcrip- tome statistical analyses were performed similarly to those already described [20, 21].

\section{Proteome study of mouse plasma}

Two-dimensional electrophoresis (2-DE) was car- ried out on plasma samples using the IPGphor Isoelectric Focusing System and the Ettan dalt six electrophoresis system (Amersham Biosciences, Little Chalfont, UK). Images of gels were digitized with a calibrated densitometer (GS-800, Bio-Rad, Hercules, CA, USA). Proteins of interest were manually excised and digested in-gel. Peptides were then identified either by peptide mass finger- printing (MALDI-TOF MS, Voyager DE-PRO in reflectron mode, Applied Biosystems, Foster City, CA, USA) or by peptide sequencing using nano(n) ESI-IT MS/MS (Finnigan LCQ IT mass spectrome- ter, ThermoQuest, Finnigan MAT, San Jose, CA, USA).

Cytokine determination in mouse liver samples

After preparation, the levels of 10 mouse cytokines and chemokines in liver homogenates were simul- taneously analysed using the MILLIPLEX" MAP Mouse Cytokine/Chemokine Magnetic Bead Panel (Millipore, Billerica, MA, USA), according to the manufacturer's instructions. The 10 cytokines and chemokines that were analysed included Ifn-c, Il-1b, Il-4, Il-6, Il-10, Il-12 (p40), Il-13, monocyte chemoattractant protein 1 (Mcp-1), macrophage inflammatory protein 1a (Mip-1a) and Tnf-a.

\section{Myeloperoxidase and leukotriene measurement in the mouse liver}

Mouse liver myeloperoxidase concentration was measured via ELISA (HK210, Hycult" Biotech, Uden, Netherlands). Leukotriene E4 is a product of the 5-lipoxygenase pathway in activated mast cells, eosinophils and monocytes. The concentra- tions were determined in the mouse livers with a commercially available enzyme immunoassay kit, the Leukotriene E4 EIA Kit (Cayman Chemical, Ann Arbor, MI, USA).

Oxidative stress analysis of the mouse liver

The concentration of 8-isoprostane, a marker of lipid peroxidation, was determined in the mouse liver samples with a commercially available enzyme immunoassay kit, the 8-Isoprostane ELISA Kit (Cayman Chemical, Ann Arbor, MI, USA). The levels of reduced glutathione (GSH) and oxidized glutathione (GSSG) were measured via capillary electrophoresis using 
the CEo- fix ${ }^{\mathrm{TM}} \mathrm{GSH}$ kit (Analis, Suarle e, Belgium) according to the manufacturer's recommendations and the P/ACE ${ }^{\mathrm{TM}}$ MDQ Capillary Electrophoresis system (Beckman Coulter, Brea, CA, USA). GSH/GSSG ratios were normalized according to the weight of the liver in each sample.

\section{French cohort of AIP patients}

Between 1974 and 2015, 602 symptomatic AIP patients were diagnosed at the French Center of Porphyria. The criteria for AIP diagnosis followed the EPNET guidelines. All patients presented with at least one acute attack that was biochemically con- firmed by the presence of a massive excretion of urinary ALA and porphobilinogen (PBG). The por- phyrin profile in urine, faeces and plasma samples allowed a differential diagnosis, eliminating both variegate porphyria and hereditary coproporphyria. The diagnosis was then confirmed by the presence of a 50\% decrease in HMBS activity in erythrocytes and/or was complemented by the identification of a causative mutation in the HMBS gene via sequenc- ing. From 1974 to 2015, a total of 46 patients were chronically affected by acute attacks and were defined as recurrent AIP patients according to the EPNET classification (four or more acute attacks for one or more years) [2]. These 46 patients required repeated IV infusions of human hemin either to treat or to prevent the occurrence of acute attacks.

\section{Human liver samples}

All procedures were performed in accordance with the 1983 and 2008 revisions of the Declaration of Helsinki, and the study was approved by the Insti- tutional Review Board and the Hospital Ethics Committee of the Bichat University Hospital. Small pieces of liver from five AIP subjects from France, Sweden and Norway were obtained (Table S1). These five patients underwent orthotopic liver transplan- tation (OLT) due to the occurrence of recurrent and debilitating attacks that were responsible for a major deterioration in quality of life. Following OLT, urinary ALA and PBG levels rapidly normal- ized, and the attacks immediately stopped. Control liver tissues were obtained at the border of liver adenoma tumours from 10 non-AIP subjects.

More details and more materials and methods are provided in the Data S1.

\section{Results}

Natural history of AIP in France since 1974

Since the establishment of the French Center of Porphyria in 1974 and through 2015, 602 symp- tomatic AIP patients with at least one acute attack of porphyria were diagnosed (of whom $81 \%$ were female). A total of $88 \%$ of patients experienced a single acute crisis, $4.4 \%$ exhibited an intermittent crisis (between one and three acute attacks/year), and $7.6 \%$ (46 patients) were disabled by repetitive acute episodes ( $\geq$ four attacks/year), most of which occurred without obvious precipitating factors.

The number of AIP patients with recurrent acute attacks increased following the introduction of haem into the pharmacopeia

In France, the administration of human hemin started in 1986. The number of AIP patients with recurrent attacks increased from 1.7\% in 1985 (four recurrent AIP patients from a total of 230 AIP patients) before the introduction of hemin to $7.5 \%$ in 2008 ( 40 recurrent AIP patients from a total of 536 AIP patients). The ratio of recurrent patients has now stabilized to $7.6 \%$ (46 recurrent AIP patients from a total of 602 AIP patients) (Fig. 1). Recurrent AIP patients already existed before the introduction of human hemin, but after its intro- duction, the frequency of recurrent AIP increased by 4.4-fold, and the interval between crises short- ened, in some cases to less than one week. Concerning the evolution of the 46 patients, 32 still exhibit recurrent AIP, of whom 18 patients receive human hemin as a preventive treatment every 7 to 14 days (before the 
appearance of any clinical symptoms), and most of them take oral opiate medication for daily pain treatment. In rare cases (n $=6$ ), it has been possible to obtain com- plete cessation of human hemin treatment.

Moreover, five patients underwent orthotopic liver transplantation (OLT) due to the presence of permanent pain and a poor quality of life (one patient died). Complete biochemical resolution was observed in the five patients after transplantation, and the four patients who are still living have remained attack-free up to now (3- to 8-year post- transplantation). On the 46 patients, a total of three patients died from unrelated diseases.

AIP mouse model and long-term treatment with hemin

To study the hepatic consequences of the chronic administration of hemin, we studied the Hmbs' mouse model, which clinically develop a peripheral neuropathy as observed in some AIP patients [19, 22]. In this model, intraperitoneal injections of phe- nobarbital-induced Alas 1 expression, resulting in a massive but transient overproduction of ALA and PBG without typical neurovisceral symptoms [19].

To reproduce recurrent attacks with frequent admin- istration of human hemin, we set up a protocol of a chronic induction of an AIP crisis treated by hemin in the AIP mouse model (Fig. 2a). PBG level was mea- sured in urines at weeks 1, 3, 6 and 8 (Fig. 2b). After the phenobarbital injection, the concentration of PBG increased and rapidly returned back to normal, as already described [23]. To further validate the biochemical induction of Alas 1 in our model, we analysed the liver expression of Alas1 using Western blot analysis. When mice were sacrificed after the last phenobarbital injection on day 3 of the eighth week (PB/Saline D3 group), we observed a 15-fold overex- pression of Alas1 when compared to noninduced mice (Saline group, Fig. 2c).

\section{Paradoxical Alas1 induction after chronic hemin infusion in the liver of Hmbs mice}

We analysed the protein expression of Alas 1 in the liver of all groups of mice sacrificed at day 5 of the eighth week. As shown in Fig. 2c, 2 days after the last phenobarbital injection (PB/Saline group), Alas1 expression returned to the basal state, but surprisingly, after repeated haem treat- ment (PB/HA group), Alas 1 expression in the liver was threefold higher than in noninduced (Saline group) and saline-treated mice (PB/Saline group).

\section{Repeated hemin injection activated $\mathrm{HOl}$ in the liver of $\mathrm{Hmbs}^{\prime}$ mice}

Overexpression of the $\mathrm{HO} 1$ gene was observed in the liver in the haem arginate-treated group compared to all other groups (Fig. 2d). A ninefold induction was identified with microarrays, and an 11-fold increase was detected via quantitative RTPCR, probably in response to the high cumulative dose of hemin. As shown by Western blot analysis, HO1 protein expression was also highly increased in the livers of mice treated with haem arginate compared to all other groups (Fig. 2e).

\section{Hepatic genome-wide transcriptomic modulation after chronic haem arginate infusion in Hmbs' mice}

The liver transcriptome of two groups of Hmbs' mice was analysed. Each group $(n=6)$ was treated sequentially for 8 weeks with phenobarbital and either treated with haem arginate (PB/HA group) or treated with saline (PB/Saline group). One hundred and fourteen probes were shown to be mod- ulated by hemin treatment when using a P-value threshold of 0.1. Among these 114 probes, 43 transcripts were down-regulated, and 50 were up- regulated (Table S2). The extent of single probe modulation ranged from a fold change of 0.01 to 29.86. To interpret the biological significance of global gene modulation, we carried out functional enrichment analysis implemented by g:Profiler software [24]. Among the nine biological processes identified in silico, four were significantly enriched in genes belonging to biological pathways linked to inflammation (Table 
S3). Interestingly, several genes involved in reactive oxygen species metabo- lism were part of the transcriptomic picture of chronic haem administration, including (i) Cybb, which encodes a component of the phagocyte oxidase enzyme complex, (ii) Rac2, which aug- ments the production of reactive oxygen species (ROS) by NADPH oxidase and (iii) glutathione Stransferase genes, which include genes such as Gstm3, Gstm4 and Gstp1. In addition, genes in the Bmp6-Id1 pathway controlling liver Hepcidin tran- scription were also induced. Altogether, these data of liver transcriptome showed that chronic hemin infusions in the mouse model significantly modi- fied biological pathways linked to inflammation, oxidative stress and iron metabolism.

Repeated haem arginate infusions induce inflammation and oxidative stress in the liver of Hmbs' mice

We further analysed a set of inflammatory and ROS markers in liver extract either at the mRNA or at the protein level. The expression of inflammatory cytokines was elevated in the liver of the mice treated by haem arginate (Table 1). The myeloperoxidase (Mpo) level was markedly increased in the haem arginate group (5.9-fold; Fig. 3a), which suggests a polynuclear neutrophil infiltration in the liver. A high leukotriene level was also observed in the liver (11.8-fold in the haem arginate group; Fig. 3a). Markers of oxidative stress were also measured in the liver of all groups of mice. In the haem arginate group, the reduced-oxidized glutathione ratio was decreased, and the level of 8-isoprostane was increased (0.3- and 12.9-fold, respectively; Fig. 3b) compared to the PB/Saline group.

To investigate the effect of chronic haem infusions at the systemic level, we analysed the plasma proteome in mice from the haem arginate group compared to the PB/Saline group. The analysis revealed that 11 plasma proteins were differentially expressed (Table S4), among which four were acute phase proteins either up-regulated (Serum amyloid P component, Complement C3 and Haptoglobin) or down-regulated (Transthyretin).

Repeated haem arginate infusions induce iron overload in the liver of Hmbs' mice

Repeated hemin injections and its degradation by $\mathrm{HO} 1$ release large amounts of iron. By measuring the liver iron content in treated mice, we showed a modest but significantly higher iron content than in nonhemin-treated mice (Figure S1a). The liver transcription profile of the mice also showed an overexpression of two genes in the haem arginate group, Bmp6 and Id1, which were first identified by microarray analysis and subsequently confirmed by qRT-PCR (Figure S1b). Bmp6 and Id1 genes were described to belong to the same pathway of hepcidin regulation [25]. This pathway is activated in response to iron overload [25].

Metabolic changes in the liver of recurrent AIP patients who receive frequent hemin administration

Explanted livers from five recurrently afflicted AIP patients were studied. All five patients received a large amount of human hemin before transplanta- tion at a frequency ranging from once a week to once a month. The last infusion before OLT took place $3 \mathrm{~h}$ before in two patients (patients P4 and P5), approximately $24 \mathrm{~h}$ before for one patient (patients P2), 3 days before in patient $\mathrm{P} 1$ and 4 days before in patient P3 (Table S1).

Histologically, liver architecture was preserved in all patients without any significant portal fibrosis or inflammation. However, an increased number of lobular inflammatory foci was observed in all cases ( $\geq 2$ foci 9200; Fig. 4a). Immunophenotypical anal- ysis demonstrated an increased number of intrasi- nusoidal MPO, CD68-positive macrophages and CD206-positive M2 macrophages in the inflamma- tory foci suggestive of inflammatory leucocytes infiltration (Fig. 5ac). 
In all explants, we observed a fourfold mean increased level of HO1 mRNA compared to mean levels from 10 control livers (Fig. 6a). At the protein level, HO1 is significantly overexpressed in the explanted livers of four patients of five. Although patients $\mathrm{P} 1$ and $\mathrm{P} 3$ did not receive hemin during the 3 to 4 days preceding surgery, HO1 was maintained at a high level.

In all explants, ALAS1 mRNA levels were in the lower range of the control values (Fig. 6a). In contrast, Western blot analyses revealed a signif- icant overexpression of ALAS1 in the liver of all AIP patients compared to controls $(\mathrm{P}=0.035$, Fig. 6a). P4 and P5 patients showed the highest induction of ALAS1.

Perls' blue staining was performed on samples from three patients and showed important iron deposits in hepatocytes and in Ku€pffer cells (Fig. 4b). Moreover, the iron status of 27 recurrent patients with frequent administration of haem arginate was collected. Median ferritin was $696 \operatorname{lg~L}^{1}$ (range: 21-2277) (Table 2), which was above the reference value in $85 \%$ of the patients. Twelve patients underwent liver magnetic reso- nance imaging; of these, 11 of them had confirmed iron overload, and the iron liver content was above $230 \operatorname{lmol~g}^{1}(\mathrm{~N}<30)$ in five patients. Twelve patients are currently being treated with serial phlebotomies or chelator administration.

\section{Discussion}

Today, the main issue in the medical care of AIP patients is the debilitating condition of patients who present with recurrent attacks. In Europe, 3\% to 5\% of individuals with AIP experienced repeated acute attacks [2]. This follow-up studies showed, for the first time, that this percentage reached $7.6 \%$ in France. This situation was rare before the introduction of human hemin, and in France, we observed a 4.4-fold increase in the number of recurrent attacks over 30 years beginning with the introduction of haem arginate to the pharma- copeia. All these patients received repeated haem arginate infusion either to treat recurrent acute crises or as a preventive treatment for recurrent attacks.

An acute porphyria attack is considered to be the consequence of an increase in hepatic ALAS1 activity that results in the accumulation of the putatively neurotoxic haem precursors ALA and PBG [3]. Hepatic ALAS1 expression is controlled via negative feedback regulation by the intracellular- free haem pool. This regulation occurs at the transcriptional level [26, 27] and mainly by the modulation of the mitochondrial translocation of ALAS1 [28]. HO1, the key enzyme in haem degradation, is one of the major determinants of the level of intracellular-free haem. Haem induces its own catabolism by inhibiting the transcriptional repres- sor BACH1, allowing the transcription of $\mathrm{HO} 1$ [29].

To date, no study has evaluated the occurrence of chronicity in AIP and experimentally explored the consequences of chronic exogenous haem therapy on the liver. We elucidate the impact of chronic haem administration on the liver in a model of Hmbs' mice. We then extended the investigations to five human-explanted livers from recurrent AIP patients.

In Hmbs' mice treated repeatedly with haem arginate, HO1 was strongly induced in the liver both at the mRNA (11-fold) and at the protein levels (6.5-fold). This induction exacerbated haem cata- bolism and secondarily increased Alas1 expres- sion. In the five explanted livers of AIP patients, HO1 mRNA expression was also increased (four- fold). These patients had received large amount of therapeutic hemin prior to OLT and have induced $\mathrm{HO} 1$ response through the BACH1/NRF2 pathway. At the protein level, HO1 is overexpressed in all patients except one. Haem arginate half-life is assumed to be 10$11 \mathrm{~h} \mathrm{[30]} \mathrm{and} \mathrm{two} \mathrm{patients,} \mathrm{P1} \mathrm{and} \mathrm{P3,} \mathrm{received} \mathrm{their} \mathrm{last} \mathrm{infusion} \mathrm{more} \mathrm{than} \mathrm{five} \mathrm{times} \mathrm{the} \mathrm{half-life.} \mathrm{Figure} \mathrm{6a} \mathrm{shows} \mathrm{that}$ although haem arginate was totally cleared from blood, the two patients exhibited a high level of HO1 induc- tion that could not be due to the last haem arginate infusion exclusively and may represent a chronic adaptation. Also, consistent with the results of the mouse study, increased expression of ALAS1 was observed chronically in all explanted livers of AIP patients, at the protein level without mRNA induction which is in favour of a post-transcrip- tional regulatory mechanism. 
Liver extracts of haem-treated mice exhibited high levels of proinflammatory cytokines Tnfa, Il-6, Il-1b and Il-12p40. This pattern is a hallmark of MyD88/TLR4 activation by haem [31]. Likewise, we observed a high expression level of S100A, S100B and Marco proteins. All these results are consistent with an M1 polarization of proinflam- matory infiltrative macrophages in the liver. Evi- dence of a proinflammatory role for haem in vivo was also observed by Wagener et al. [32] after a single-dose hemin infusion into the tail veins of BALB/c wild-type mice, leading to the infiltration of leukocytes in the liver. Unlike our protocol, this study utilized a single high dose of hemin, whereas in the present study, we investigated the effects of a cumulative dose of hemin over a 2-month period and observed chronic and complex inflammatory lesions.

The liver extracts from haem-treated mice also showed an increased expression of Mrc1, Mgl1 and the Il-10 antiinflammatory cytokines, which were suggestive of M2 polarization of macrophages.

Human-explanted livers from AIP patients also highly expressed MRC1 (CD206), which was com- patible with a M2 polarization. One distinguishing characteristic between M2 and M1 macrophage polarization is the intracellular redox status [33]. The oxidation status observed in liver extracts of haem-treated mice is characteristic of an M2 polarization (a reduced GSH/GSSG ratio, a high level of 8-isoprostane and an induction of Cybb and Rac2; Fig. 3b and Table S2). Altogether, these data support the idea of a complex inflammatory pattern consisting of an M1/Th1 proinflammatory pattern associated with a predominant M2/Th2 anti-inflammatory response, according to the oxi- dation status. Several studies showed that the antiinflammatory cytokine Il-10 induces HO1 [34-36] which could amplify the effects of haem per se on HO1 expression.

Also, notable was the observation of the presence of iron deposition in livers of both haem-treated mice and AIP patients exposed to chronic haem infu- sions (Figures S1 and $4 \mathrm{~b}$ ). Repeated hemin infu- sion is responsible for a high cumulative dose of iron (Table 2). Marsden et al. [15] have shown that there was a significant correlation between the serum ferritin concentration and number of doses that were administered. In the explanted livers,

iron overload was observed both in hepatocytes and in Ku€pffer cells where it presented as large aggregates. In fact, Vinchi et al. [37] showed that during intravascular hemolysis leading to free haem excess, the liver is the most susceptible organ to haem overload, and when hemopexin capacity is exceeded, haem accumulates mainly in Ku€pffer cells. In line with our results, three well- documented studies concerning recurrent AIP patients reported also that regular hemin infusions trigger variable liver iron overload $[8,9,38]$.

Different studies have shown that iron overload impairs the hepatic mitochondrial metabolism [39, 40]. High amount of free iron released by HO-1 catalyses the formation of reactive oxygen species and is responsible for alteration of mitochondrial respiration in rodent submitted to systemic inflam- mation [41]. Thus, liver iron overload, as observed in recurrent AIP patient, could worsen the impair- ment of the respiratory chain and the tricarboxylic acid cycle of the liver mitochondrias during an acute attack, as previously shown in the AIP mouse model [42].

Oxidative stress, as observed in Fig. 3b, induces the dissociation of the stress-sensitive KEAP1 pro- tein from NRF2. In turn, NRF2 translocates to the nucleus and then transactivates HO1 expression through the AREs sequences and consequently enhances endogenous free haem degradation [43].

\section{Conclusion}

AIP is considered as an intermittent disease, but $7.6 \%$ of patients have a chronic life-threatening debilitating condition. Our study highlights a key role of hemin infusions in the pathophysiology of AIP chronic attacks. Nevertheless, hemin is the most effective treatment for acute neurovisceral attacks and is life-saving. The introduction of haem arginate decreased mortality at a price of increas- ing recurrent attacks. Hemin treatment needs there- fore to be restricted to patients with severe forms of 
AIP crises with the occurrence of progressive signs of a central or peripheral neurological alteration, pro- found hyponatremia or hyperalgesic acute crisis unresponsive to symptomatic treatment. Altogether, we bring convergent arguments showing that chronic exogenous haem infusions were responsi- ble for a physiological response which activates chronically HO1 expression. The three factors that could be implicated in HO1 induction are haem per se, the inflammation characterized by an adaptive M2 macrophage polarization promoting the IL-10- HO1 amplifying loop and the oxidative stress generated by iron overload (Fig. 6b). In the context of AIP, exacerbated free haem degradation would reduce the free haem pool, which in turn would up- regulate ALAS1, promote ALA and PBG overpro- duction and finally, exacerbate porphyric symp- toms. In humans, the HO1 promoter contains a (GT)(n) dinucleotide repeat that is related to HO1 expression and activity, and it would be tempting to speculate that this polymorphism could modu- late the basal level of the free haem pool and in turn the penetrance and the severity of AIP. The present data that demonstrate the role of chronic inflam- mation on $\mathrm{HO} 1$ activation after repeated haem therapy is crucial for the improvement of recurrent AIP patient care. Subsequent development of new and safe ALAS1 and/or HO1 inhibitors would greatly improve AIP treatment.

\section{Conflict of interest statement}

LG, JCD, PH, ES and AKA were funded for attend- ing meeting related to ongoing clinical trial by Alnylam Pharmaceuticals. Other authors have no conflict of interest to declare.

\section{Acknowledgements}

We are very grateful to Professor Urs Meyer and Doctor Anne-Kathrin Peyer for having provided the living Hmbs' mice, to Eric Couchi for his excel- lent supervision of animal breeding and treatments and to Sylvie Simonin for urinary ALA and PBG measurements.

\section{References}

1 Puy H, Gouya L, Deybach JC. Porphyrias. Lancet 2010; 375: 924-37.

2 Elder G, Harper P, Badminton M, Sandberg S, Deybach JC. The incidence of inherited porphyrias in Europe. J Inherit Metab Dis 2013; 36: 849-57.

3 Meyer UA, Schuurmans MM, Lindberg RL. Acute porphyrias: pathogenesis of neurological manifestations. Semin Liver Dis 1998; 18: 43-52.

4 Sima AA, Kennedy JC, Blakeslee D, Robertson DM. Experi- mental porphyric neuropathy: a preliminary report. Can J Neurol Sci 1981; 8: 105-13.

5 Soonawalla ZF, Orug T, Badminton MN, et al. Liver trans- plantation as a cure for acute intermittent porphyria. Lancet 2004; 363: 705-6.

6 Wahlin S, Harper P, Sardh E, Andersson C, Andersson DE, Ericzon BG. Combined liver and kidney transplanta- tion in acute intermittent porphyria. Transpl Int 2010; 23: e18-21.

7 Dar FS, Asai K, Haque AR, Cherian T, Rela M, Heaton N. Liver transplantation for acute intermittent porphyria: a viable treatment? Hepatobiliary Pancreat Dis Int 2010; 9: 93-6.

8 Dowman JK, Gunson BK, Mirza DF, Bramhall SR, Badminton MN, Newsome PN. Liver transplantation for acute intermittent porphyria is complicated by a high rate of hepatic artery thrombosis. Liver Transpl 2012; 18: 195-200.

9 Yasuda M, Erwin AL, Liu LU, et al. Liver transplantation for acute intermittent porphyria: biochemical and pathologic studies of the explanted liver. Mol Med $2015 ; 21: 487-95$.

10 Dowman JK, Gunson BK, Bramhall S, Badminton MN, Newsome PN. Liver transplantation from donors with acute intermittent porphyria. Ann Intern Med 2011; 154: 571-2. 
11 Handschin C, Lin J, Rhee J, et al. Nutritional regulation of hepatic heme biosynthesis and porphyria through PGC- 1alpha. Cell 2005; 122 : 505-15.

12 Hift RJ, Meissner PN. An analysis of 112 acute porphyric attacks in Cape Town, South Africa: Evidence that acute intermittent porphyria and variegate porphyria differ in susceptibility and severity. Medicine (Baltimore) 2005; 84: 48-60.

13 Anderson KE, Collins S. Open-label study of hemin for acute porphyria: clinical practice implications. Am J Med 2006; 119: 801.e819-24.

14 Bonkovsky HL, Maddukuri VC, Yazici C, et al. Acute por- phyrias in the USA: features of 108 subjects from porphyrias consortium. Am J Med 2014; 127: $1233-41$.

15 Marsden JT, Guppy S, Stein P, et al. Audit of the use of regular haem arginate infusions in patients with acute porphyria to prevent recurrent symptoms. JIMD Rep 2015; 22: 57-65.

16 Pischik E, Kauppinen R. An update of clinical management of acute intermittent porphyria. Appl Clin Genet 2015; 8: $201-14$

17 Stein P, Badminton M, Barth J, Rees D, Stewart MF. Best practice guidelines on clinical management of acute attacks of porphyria and their complications. Ann Clin Biochem 2013; 50: 217-23.

18 Naik HSM, Sanderson SC, Balwani M, Desnick RJ. Experi- ences and concerns of patients with recurrent attacks of acute hepatic porphyria: A qualitative study. Mol Genet Metab 2016; 119: 278-83.

19 Lindberg RL, Porcher C, Grandchamp B, et al. Porphobilino- gen deaminase deficiency in mice causes a neuropathy resembling that of human hepatic porphyria. Nat Genet 1996; 12: 195-9.

20 Diamond D, Krasnoselsky A, Burnum K, et al. Proteome and computational analyses reveal new insights into the mecha- nisms of hepatitis C virus-mediated liver disease posttrans- plantation. Hepatology 2012; 56: 28-38.

21 Rasmussen A, Tchitchek N, Susnow N, et al. Early transcrip- tional programming links progression to hepatitis C virus- induced severe liver disease in transplant patients. Hepatol- ogy 2012; 56: 17-27.

22 Lindberg RL, Martini R, Baumgartner M, et al. Motor neu- ropathy in porphobilinogen deaminase-deficient mice imi- tates the peripheral neuropathy of human acute porphyria. J Clin Invest 1999; 103: 1127-34.

23 Schuurmans MM, Hoffmann F, Lindberg RL, Meyer UA. Zinc mesoporphyrin represses induced hepatic 5-aminolevulinic acid synthase and reduces heme oxygenase activity in a mouse model of acute hepatic porphyria. Hepatology 2001; 33: 1217-22.

24 Reimand J, Kull M, Peterson H, Hansen J, Vilo J. g:Profiler-a web-based toolset for functional profiling of gene lists from large-scale experiments. Nucleic Acids Res 2007; 35: W193-200

25 Kautz L, Meynard D, Monnier A, et al. Iron regulates phos- phorylation of Smad1/5/8 and gene expression of Bmp6, Smad 7, Id1, and Atoh8 in the mouse liver. Blood 2008; 112: 1503-9.

26 Gotoh S, Nakamura T, Kataoka T, Taketani S. Egr-1 regulates the transcriptional repression of mouse delta-aminolevulinic acid synthase 1 by heme. Gene $2011 ; 472: 28-36$.

27 Yamamoto M, Kure S, Engel JD, Hiraga K. Structure, turn- over, and heme-mediated suppression of the level of mRNA encoding rat liver deltaaminolevulinate synthase. J Biol Chem 1988; 263: 15973-9.

28 Lathrop JT, Timko MP. Regulation by heme of mitochondrial protein transport through a conserved amino acid motif. Science 1993; $259: 522-5$.

29 Sun JHH, Takaku K, Nakajima O, et al. Hemoprotein Bach1 regulates enhancer availability of heme oxygenase-1 gene. EMBO J 2002; $21: 5216-24$.

30 Tokola O, Tenhunen R, Volin L, Mustajoki P. Pharmacoki- netics of intravenously administered haem arginate. Br J Clin Pharmacol 1986; 22: 331-5.

31 Figueiredo RT, Fernandez PL, Mourao-Sa DS, et al. Charac- terization of heme as activator of Toll-like receptor 4. J Biol Chem 2007; 282: 20221-9. 
32 Wagener FA, Eggert A, Boerman OC, et al. Heme is a potent inducer of inflammation in mice and is counteracted by heme oxygenase. Blood 2001; 98 : $1802-11$.

33 Dobashi K, Aihara M, Araki T, et al. Regulation of LPS induced IL-12 production by IFN-gamma and IL-4 through intracellular glutathione status in human alveolar macro- phages. Clin Exp Immunol 2001; 124: 290-6.

34 Lee TS, Chau LY. Heme oxygenase-1 mediates the anti- inflammatory effect of interleukin-10 in mice. Nat Med 2002; 8: 240-6.

35 Chen S, Kapturczak MH, Wasserfall C, et al. Interleukin 10 attenuates neointimal proliferation and inflammation in aor- tic allografts by a heme oxygenasedependent pathway. Proc Natl Acad Sci U S A 2005; 102: 7251-6.

36 Ricchetti GA, Williams LM, Foxwell BM. Heme oxygenase 1 expression induced by IL-10 requires STAT-3 and phospho- inositol-3 kinase and is inhibited by lipopolysaccharide. J Leukoc Biol 2004; 76: 719-26.

37 Vinchi F, Gastaldi S, Silengo L, Altruda F, Tolosano E. Hemopexin prevents endothelial damage and liver congestion in a mouse model of heme overload. Am J Pathol 2008; 173: 289-99.

38 Willandt B, Langendonk JG, Biermann K, et al. Liver fibrosis associated with iron accumulation due to long-term heme- arginate treatment in acute intermittent porphyria: a case series. JIMD Rep 2015; 25: 77-81.

39 Bacon B, Park C, Brittenham G, O’Neill R, Tavill A. Hepatic mitochondrial oxidative metabolism in rats with chronic dietary iron overload. Hepatology 1985; 5: 789-97.

40 Volani C, Doerrier C, Demetz E, et al. Dietary iron loading negatively affects liver mitochondrial function. Metallomics 2017; 9: 1634-44.

41 Duvigneau J, Piskernik C, Haindl S, et al. A novel endotoxin- induced pathway: upregulation of heme oxygenase 1, accu- mulation of free iron, and free ironmediated mitochondrial dysfunction. Lab Invest 2008; 88: 70-7.

42 Homedan C, Laafi J, Schmitt C, et al. Acute intermittent porphyria causes hepatic mitochondrial energetic failure in a mouse model. Int J Biochem Cell Biol 2014; 51: 93-101.

43 Naito Y, Takagi T, Higashimura Y. Heme oxygenase-1 and anti-inflammatory M2 macrophages. Arch Biochem Biophys 2014; $564: 83-8$.

Correspondence: Laurent Gouya, Centre Franc"ais des Porphyries, Ho^pital Louis Mourier, AP-HP, 178 rue des Renouillers, 92701 Colombes, France. (fax: +33 1476067 03; e-mail: laurent.gouya@inserm.fr). 
